\title{
The mitochondrial transport protein SLC25A43 affects drug efficacy and drug-induced cell cycle arrest in breast cancer cell lines
}

\author{
MARIKE GABRIELSON ${ }^{1}$ and ELISABET TINA ${ }^{1,2}$ \\ ${ }^{1}$ School of Health and Medical Sciences, Örebro University Hospital; \\ ${ }^{2}$ Clinical Research Centre, Örebro University Hospital, SE-70185 Örebro, Sweden
}

Received November 6, 2012; Accepted December 10, 2012

DOI: $10.3892 /$ or.2013.2247

\begin{abstract}
The mitochondria have been identified as key players of apoptosis, cell proliferation and cell cycle regulation. However, the role of mitochondria in breast cancer and treatment failure remains unclear. We have previously shown a common deletion of the gene SLC25A43 in human epidermal growth factor receptor 2 (HER2)-positive breast cancer. This gene is coding for a mitochondrial inner membrane transporter and, to date, little is known about the function of this protein. We have also found that low protein expression of SLC25A43 significantly correlates with a lower S phase fraction in HER2-positive breast cancer. The aim of this study was to investigate whether knockdown (KD) of $S L C 25 A 43$ could have an effect on the cytotoxicity of different cytostatic drugs using MCF10A, MCF7 and BT-474 cells. Following siRNA-mediated KD of SLC25A43, one non-malignant and two breast cancer cell lines were exposed to the anthracycline epirubicin or the taxane paclitaxel. The HER2-positive breast cancer cells were also exposed to the targeted therapy trastuzumab and dual exposure to trastuzumab and paclitaxel. We found that KD of SLC25A43 resulted in a decreased cytotoxic effect of paclitaxel in the two cancer cell lines $(\mathrm{P}<0.05)$. Further analysis of cell cycle phase distribution showed that $\mathrm{KD}$ increased the paclitaxel-induced $\mathrm{G} 2 / \mathrm{M}$ block in these two cell lines $(\mathrm{P}<0.05)$. KD of SLC25A43 also reduced the inhibitory effect of trastuzumab on cell proliferation in the HER2-positive cancer cell line BT-474 $(\mathrm{P}<0.05)$, and the drug-induced G0/G1 block $(\mathrm{P}<0.05)$. Moreover, SLC25A43 influenced the percentage of Ki-67-positive cells. Our findings demonstrate that the mitochondrial protein SLC25A43 affects drug efficacy and cell cycle regulation following drug exposure in breast cancer cell lines.
\end{abstract}

Correspondence to: Dr Marike Gabrielson, School of Health and Medical Sciences, Örebro University Hospital, SE-70185 Örebro, Sweden

E-mail: marike.gabrielsson@oru.se

Key words: SLC25A43, cytotoxicity, viability, cell cycle arrest, human epidermal growth factor receptor 2-positive breast cancer

\section{Introduction}

Although a number of cytostatic drugs with different mechanisms of action are used in the treatment of breast cancer, intrinsic or acquired resistance is a common problem culminating in the treatment failure (1). Taxanes and anthracyclines are widely used and their antitumour activity contributes to apoptosis in cancer cells through interaction via several intracellular functions $(2,3)$. For instance, the taxane paclitaxel exerts its apoptotic effects by stabilising the microtubuli thus preventing them from disassembling (4). It is also a potent inhibitor of chromosomal replication resulting in late Gap 2 (G2) or mitotic (M) block of the cell cycle (5). Paclitaxel is generally used as a single agent as well as in combination therapy with other chemotherapeutic agents to treat early and advanced stage breast cancer (6). The reported overall response rate of paclitaxel is 21 to $54 \%$ when used as a firstline single agent therapy. Anthracyclines such as epirubicin, on the other hand, act in part by inhibiting DNA and RNA synthesis as well as by inducing permanent double strand DNA breaks by inhibiting the enzyme topoisomerase II thereby promoting G2-blockage (7,8). Anthracycline-based polychemotherapy reportedly reduces the mortality rate by 20 to $38 \%$ (9). However, anthracyclines are associated with a higher rate of acute and late adverse effects, such as increased risk of cardiotoxicity and secondary leukaemia (10).

In order to achieve an individualised antitumour therapy, targeted therapy directed against specific cellular molecules has been developed. The monoclonal antibody trastuzumab is the first targeted therapy directed against the extracellular domain of the human epidermal growth factor receptor 2 (HER2). Trastuzumab is often used in treatment of patients with an amplification/overexpression of HER2. Binding of trastuzumab to the HER2-receptor induces a G1 block, thus reducing both the proliferation and survival advantages of the tumours (11-15). Trastuzumab has a modest overall response rate ranging from 15 to $30 \%$ when used as a neoadjuvant single agent (16). In clinical settings, trastuzumab is predominantly administered in combination with chemotherapy drugs such as taxanes to improve disease-free and overall survival (17-19). Nevertheless, even when combining targeted therapy and chemotherapy, treatment failure remains an ongoing problem 
in the clinic, indicating the complexity of the mechanisms involved in drug efficiency.

In recent years, a hypothesis has emerged, identifying a new role of the mitochondria as an important regulator of the cell cycle by connecting mitochondrial function and energetic status with cell cycle progression (20-26). This supports the theory that altered mitochondrial function along with deregulated energy metabolism are key for cancer cells to sustain uncontrolled cell proliferation and avoid apoptosis (27).

We previously described novel findings of a commonly deleted gene in HER2-positive breast cancer (28). This gene encodes the mitochondrial inner membrane solute carrier protein SLC25A43. However, the complete function of SLC25A43 along with the substrate carried by this membrane transporter remains unknown. We noted that lower expression of SLC25A43 in the tumours correlated significantly with a lower S phase fraction in HER2-positive breast cancer (28).

The aim of this study was to investigate the possible role of the mitochondrial protein SLC25A43 in drug sensitivity in vitro. Our results showed that knockdown (KD) of SLC25A43 in different breast cell lines altered the sensitivity to the cytostatic drugs, as demonstrated by altered cell viability and altered distribution and regulation of cell cycle phases. The findings presented herein support the theory of a mitochondrial role in drug susceptibility.

\section{Materials and methods}

Cell culturing. The immortalized breast epithelial cell line MCF10A, the HER2-negative breast adenocarcinoma cell line MCF7 and the HER2-positive breast cancer cell line BT-474 were all obtained from the American Type Culture Collection (Manassas, VA, USA). MCF10A was cultured in D-MEM/F-12 supplemented with $10 \% \mathrm{FBS}, 10 \mu \mathrm{g} / \mathrm{ml}$ insulin, $20 \mathrm{ng} / \mathrm{ml}$ $\mathrm{H}-\mathrm{EGF}$ and $0.5 \mu \mathrm{g} / \mathrm{ml}$ hydrocortisone. MCF7 was cultured in Eagle's minimum essential medium (MEM) supplemented with $10 \%$ FBS and $10 \mu \mathrm{g} / \mathrm{ml}$ insulin, and BT-474 was cultured in RPMI-1640 supplemented with $10 \%$ FBS and $10 \mu \mathrm{g} / \mathrm{ml}$ insulin. Cells were cultured in a humidified atmosphere at $37^{\circ} \mathrm{C}$ with $5 \% \mathrm{CO}_{2}$.

The cells were seeded at a density of $25 \times 10^{3}$ cells $/ \mathrm{cm}^{2}$, $24 \mathrm{~h}$ before transfection. Transfection was performed using Lipofectamine $^{\mathrm{TM}} 2000$ (Invitrogen, Carlsbad, CA, USA) and scrambled siRNA (siCtrl) or target-specific siRNA [Hs_LOC203427_2 (Qiagen Sciences, Germantown, MD, USA)] (siSLC) for KD, according to the manufacturer's recommendations. The obtained SLC25A43 mRNA KD was $90 \%$ in MCF10A, 90\% in MCF7 and 75\% in BT-474, and was stable in all cell lines for a minimum of $96 \mathrm{~h}$.

For all cytotoxicity assays, cells were exposed to the drugs $24 \mathrm{~h}$ after transfection and incubated for $72 \mathrm{~h}$, using 16 or $160 \mathrm{nM}$ paclitaxel or 2.5 or $10 \mu \mathrm{M}$ epirubicin (Actavis, Hafnarfjordur, Iceland). BT-474 cells were also subjected to exposure of 10 or $100 \mu \mathrm{g} / \mathrm{ml}$ trastuzumab (Roche $\mathrm{AB}$, Stockholm, Sweden) or a combination of trastuzumab $(10 \mu \mathrm{g} / \mathrm{ml})$ and paclitaxel $(16 \mathrm{nM})$, referred to as T/P. As a drug-free control for all experiments, cells were transfected and cultured in medium without cytostatic drugs. Incubation with epirubicin was terminated after $1 \mathrm{~h}$ by replacing the medium with fresh medium.

\section{Flow cytometry assays}

Determination of viable cells. Cell viability was determined by incubating collected cells in the culture medium together with the trypsinized cells using $0.25 \mu \mathrm{g}$ 7-AAD (BD Biosciences, San Jose, CA, USA) for $10 \mathrm{~min}$ at room temperature and protected from light, according to the manufacturer's protocol.

Inhibition of cell proliferation assay. Measurement of cell proliferation was carried out using PKH67 Green Fluorescent Cell Linker (Sigma-Aldrich, St. Louis, MO, USA) according to the manufacturer's protocol. PKH67 is a green fluorochrome that incorporates into the cell membrane without affecting cell viability. Following cell division, fluorescence intensity is decreased due to dilution of the fluorochrome. The cells were stained with PKH67 at time of seeding.

Cell cycle phase analysis. Analysis of cell cycle phase distribution was performed as previously described (29) on isolated cell nuclei using $100 \mu \mathrm{g} / \mathrm{ml}$ propidium iodide (PI) (SigmaAldrich) for DNA-staining.

Cell cycle regulation assay with $\mathrm{Ki}-67$ and $p 21$. The expression of $\mathrm{Ki}-67$ and p21 was analysed after $72 \mathrm{~h}$ of exposure with $16 \mathrm{nM}$ paclitaxel, $2.5 \mu \mathrm{M}$ epirubicin, $10 \mu \mathrm{g} / \mathrm{ml}$ trastuzumab or a combination of trastuzumab $(10 \mu \mathrm{g} / \mathrm{ml})$ and paclitaxel $(16 \mathrm{nM})$, as indicated. Pelleted cells were resuspended for $10 \mathrm{~min}$ with an ice cold lysing solution containing $0.1 \%$ Igepal CA-630 in wash buffer (1\% FBS in PBS) to isolate cell nuclei. The nuclei were then washed once with ice cold Wash buffer before adding antibodies against p21 Alexa Fluor ${ }^{\circledR} 488$ (1:50, clone 12D1; Cell Signaling Technology, Inc., Danvers, MA, USA) and $\mathrm{Ki}-67 \mathrm{PE}$ (clone 56; BD Biosciences) to one tube and isotype control to a second tube [IgG isotype for Alexa Fluor 488 (1:50) and IgG1 isotype for PE]. All tubes were supplemented with $0.5 \mu \mathrm{g}$ 7-AAD (BD Biosciences) for DNA staining and incubated for $15 \mathrm{~min}$. The nuclei were then diluted with PBS and stored on ice prior to analysis. All incubation steps were performed on ice.

The flow cytometry analyses were performed $96 \mathrm{~h}$ after transfection using an EPICS Altra equipped with an argon laser (488 nm) and EXPO 32 software (Beckman-Coulter, Fullerton, CA, USA). During acquisition, 10,000 events were collected. For analysis of cell viability, PKH67 and the expression of cell cycle proteins, Kaluza software (Beckman-Coulter) was used. Cell cycle phase distribution was analysed using ModFit LT v3.2 (Verity Software House, USA). To analyse the expression of $\mathrm{Ki}-67$ and p21 a gate was set on the 7-AAD histogram to exclude sub-G0/G1 events and then the isotype control was used to determine the cut-off for positivity. The positivity was expressed as a percentage of positive cells.

Statistical analysis. The Mann-Whitney test was used to assess statistical significance in all assays. $\mathrm{P}<0.05$ was considered to indicate a statistically significant difference. SPSS 17.0 statistical software for Windows (SPSS Inc., Chicago, IL, USA) was used for all tests.

\section{Results}

Reduced paclitaxel efficacy following SLC25A43 knockdown. Our experiments described the effects of SLC25A43 on drug-related cellular outcome and sensitivity utilising siRNA- 

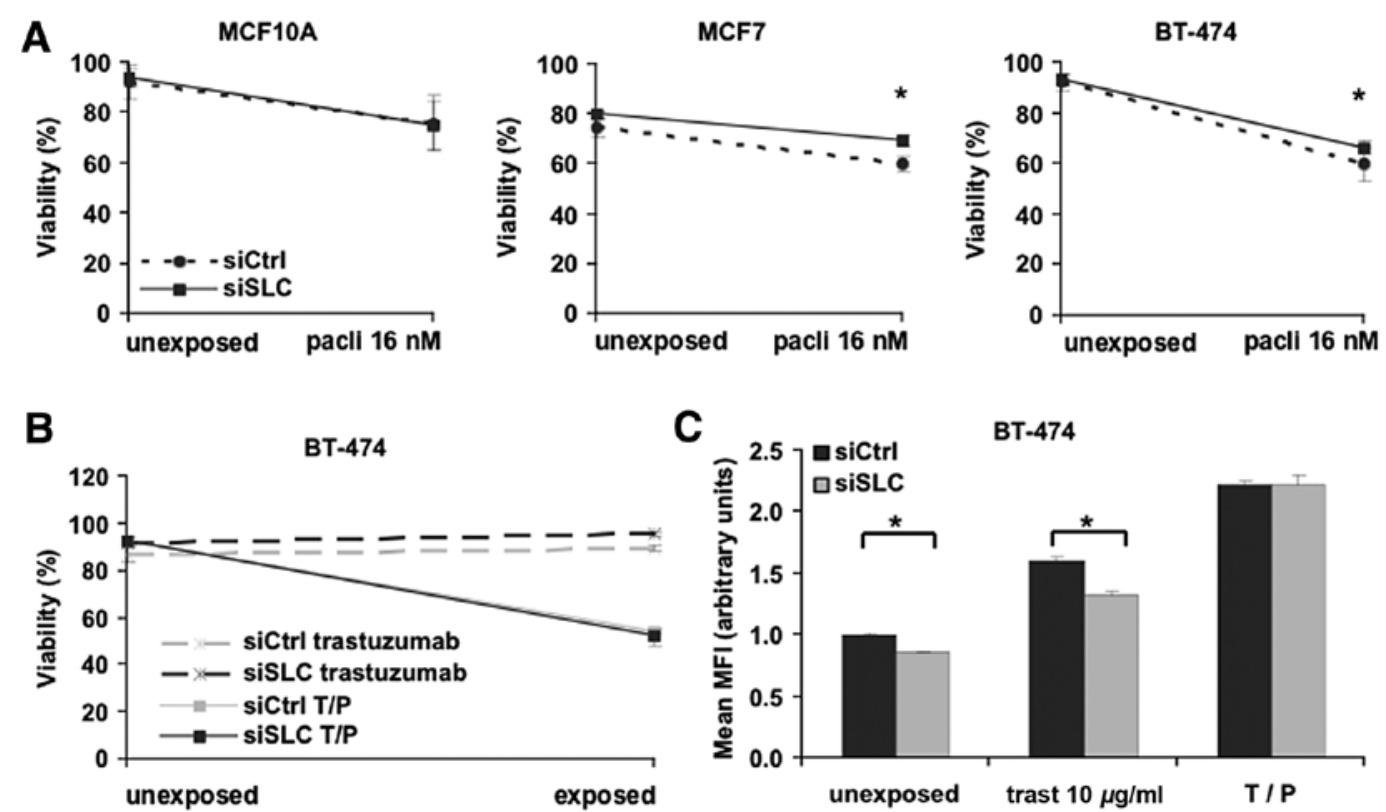

Figure 1. SLC25A43 KD reduces the cytotoxic effect of paclitaxel and trastuzumab in breast cancer cells. (A) KD of $S L C 25 A 4324 \mathrm{~h}$ before exposing cells to $16 \mathrm{nM}$ paclitaxel for $72 \mathrm{~h}$ significantly increased viability in the MCF7 and BT-474 cancer cells but not in the non-malignant mammary epithelial cells MCF10A, compared to control siRNA. (B) Viability of BT- 474 with or without $S L C 25 A 43 \mathrm{KD}$ after $72 \mathrm{~h}$ exposure with $16 \mathrm{nM}$ paclitaxel, $10 \mu \mathrm{g} / \mathrm{ml}$ trastuzumab or combination of paclitaxel and trastuzumab (T/P) $(16 \mathrm{nM}$ and $10 \mu \mathrm{g} / \mathrm{ml})$. Viability after T/P exposure was independent of $S L C 25 A 43 \mathrm{KD}$. (C) Trastuzumabinduced inhibition of cell proliferation, measured using PKH67, was significantly reduced after SLC25A43 KD. However, when combining trastuzumab and paclitaxel $(16 \mathrm{nM}$ and $10 \mu \mathrm{g} / \mathrm{ml})$, the drug-induced inhibition was independent of $S L C 25 A 43 \mathrm{KD}$. For all assays, three experiments were performed in duplicates. The data are expressed as the means \pm SD. ${ }^{*} \mathrm{P}<0.05$.

mediated mRNA KD in in vitro models. The non-malignant cell line MCF10A and the two cell lines MCF7 and BT-474, with different clinically relevant breast cancer phenotypes, were used. To evaluate the effects of $S L C 25 A 43 \mathrm{KD}$ on cytotoxicity, we analysed the viability of MCF10A, MCF7 and BT-474 cells after 72-h exposure to paclitaxel or epirubicin. The sensitivity for paclitaxel was not altered in MCF10A cells after KD of $S L C 25 A 43$ (Fig. 1A). By contrast, KD of SLC25A43 resulted in a significant reduction of cytotoxic effects by $16 \mathrm{nM}$ paclitaxel in the two breast cancer cell lines MCF7 and BT-474 compared to siCtrl (Fig. 1A). The reduction in cytotoxicity was also observed at the higher concentration of paclitaxel in MCF7 cells $(\mathrm{P}<0.05)$. Contrary to the paclitaxel exposure, KD of SLC25A43 did not influence the cytotoxic effect of epirubicin in any cell line (data not shown).

The HER2-positive breast cancer cell line, BT-474, was further investigated through exposure with the targeting drug trastuzumab. Trastuzumab alone does not directly induce cell death in vitro; however, it inhibits proliferation and is an important agent when treating HER2-positive breast tumours (30). We therefore measured both the viability and inhibition of proliferation of BT-474 cells subjected to exposure of either trastuzumab $(10 \mu \mathrm{g} / \mathrm{ml})$ or a combination-dose of trastuzumab $(10 \mu \mathrm{g} / \mathrm{ml})$ and paclitaxel $(16 \mathrm{nM})(\mathrm{T} / \mathrm{P})$ (Fig. 1B and C). As expected, trastuzumab alone did not induce cell death (Fig. 1D). However, the reduced cytotoxic effect of paclitaxel after $S L C 25 A 43 \mathrm{KD}$ was no longer present when paclitaxel was combined with trastuzumab. Measuring proliferation of BT-474 cells after trastuzumab exposure revealed that SLC25A43 KD contributes to a significantly lower inhibition of cell proliferation (Fig. 1C). This effect was, however, eliminated when combining trastuzumab and paclitaxel. These data show that KD of SLC25A43 influences the efficacy of paclitaxel and trastuzumab in the breast cancer cell lines.

SLC25A43 affects the cell cycle phase distribution upon drug exposure. Paclitaxel, epirubicin and trastuzumab are all known to induce cell cycle arrest, thus leading to cell death or inhibition of proliferation. To evaluate a possible influence of SLC25A43 KD on the cell cycle, we analysed the cell cycle phase distribution in surviving cells following drug exposure.

Paclitaxel blocks the cells in the late $\mathrm{G} 2$ and $\mathrm{M}$ phases of the cell cycle (5), and, as shown in Fig. 2A-C, exposure to paclitaxel induced a G2/M block in all three cell lines. Notably, SLC25A43 KD resulted in a significantly higher G2/M block at $16 \mathrm{nM}$ paclitaxel in MCF7 and BT-474 compared to control. This, however, was not seen in MCF10A cells. The significant difference in $\mathrm{G} 2 / \mathrm{M}$ distribution remained in MCF7 $(\mathrm{P}<0.05)$ and BT-474 $(\mathrm{P}<0.05)$ cells at the higher concentration of paclitaxel.

Epirubicin induces permanent double strand breaks leading to $\mathrm{G} 2 / \mathrm{M}$ block $(7,8)$. Similar to paclitaxel, epirubicin also induced G2/M block in the cell lines upon exposure (Fig. 2A-C). However, only MCF7 cells showed a significant change in cell cycle phase distribution between siCtrl and $S L C 25 A 43 \mathrm{KD}$ at the lower concentration $(2.5 \mu \mathrm{M})$ of epirubicin. Exposure of the cell lines to $10 \mu \mathrm{M}$ of epirubicin resulted in less $\mathrm{G} 2 / \mathrm{M}$ block compared to $2.5 \mu \mathrm{M}$, but there was a significantly higher fraction of cells in G2/M phase in SLC25A43 KD compared to control in both MCF7 $(\mathrm{P}<0.05)$ and BT-474 $(\mathrm{P}<0.05)$ cells.

Next, we examined the effects of trastuzumab on the cell cycle phase distribution after KD of $S L C 25 A 43$. Trastuzumab is known to exhibit growth arrest in the G1 phase, partly due to inhibition of proteins involved in regulating the cell 

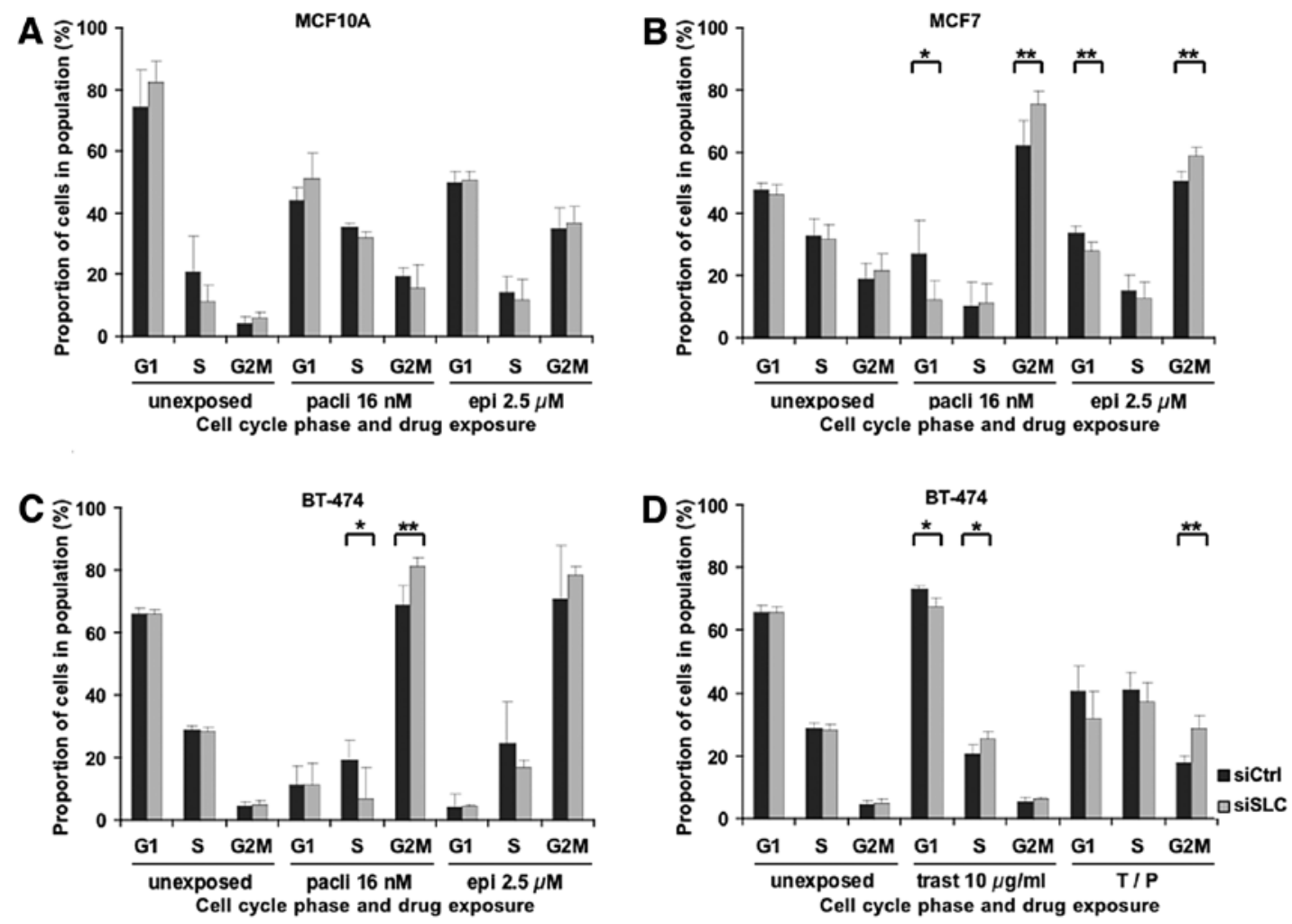

Figure 2. SLC25A43 KD significantly alters the cell cycle phase distribution after drug exposure in the breast cancer cell lines. (A-C) Cell cycle phase distribution in (A) MCF10A, (B) MCF7 and (C) BT-474 cells after $16 \mathrm{nM}$ paclitaxel or $2.5 \mu \mathrm{M}$ epirubicin exposure for $72 \mathrm{~h}$, comparing SLC25A43 KD with control siRNA. Both paclitaxel and epirubicin induced G2M block in all three cell lines, and KD of SLC25A43 significantly increased the paclitaxel-induced block in MCF7 and BT-474 cells. Only in MCF7 did SLC25A43 KD significantly alter the epirubicin-induced G2M block. (D) Cell cycle phase distribution in BT-474 after $72 \mathrm{~h}$ of $10 \mu \mathrm{g} / \mathrm{ml}$ trastuzumab or trastuzumab-paclitaxel double exposure (T/P) (16 nM and $10 \mu \mathrm{g} / \mathrm{ml})$, comparing SLC25A43 KD with control siRNA. Exposure with trastuzumab induced a G1 block that was significantly reduced in $S L C 25 A 43 \mathrm{KD}$ cells. SLC25A43 KD prior to double exposure significantly increased the paclitaxel-induced G2/M block. For all assays, three experiments were performed in duplicates. The data are expressed as the means $\pm \mathrm{SD}$. $\mathrm{P}<0.05$ and ${ }^{* *} \mathrm{P}<0.01$.
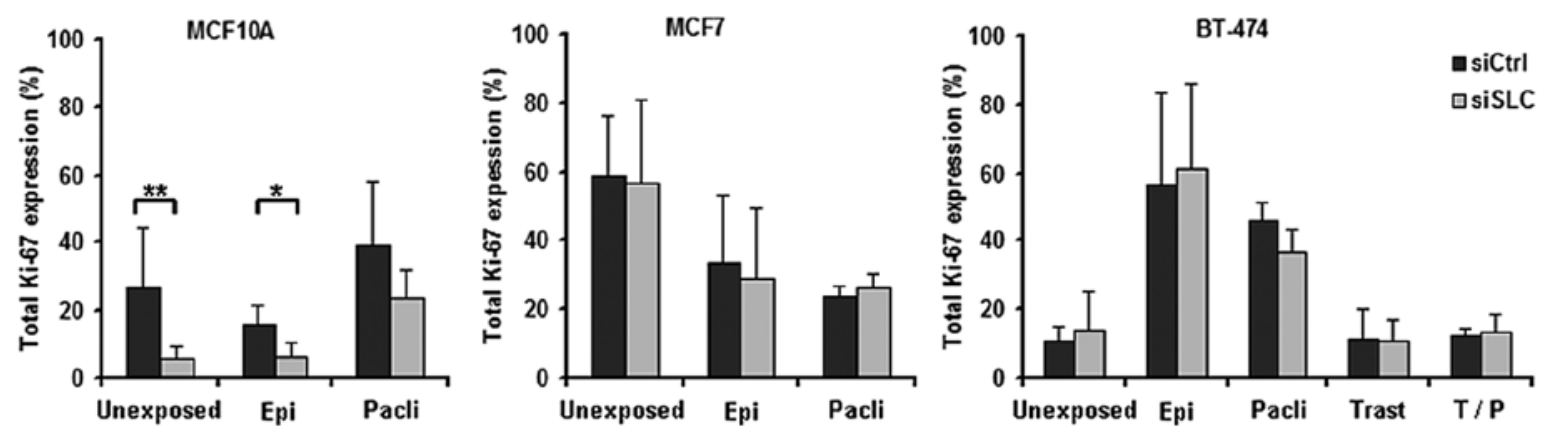

Figure 3. SLC25A43 KD reduces the expression of proliferation marker Ki-67 in non-malignant MCF10A cells after epirubicin exposure. Total Ki-67 expression in MCF10A, MCF7 and BT-474 cells $72 \mathrm{~h}$ after exposure with $16 \mathrm{nM}$ paclitaxel or $2.5 \mu \mathrm{M}$ epirubicin, comparing siCtrl and siSLC-transfected cells BT-474 cells were also exposed to $10 \mu \mathrm{g} / \mathrm{ml}$ trastuzumab or trastuzumab-paclitaxel double exposure (T/P) $(16 \mathrm{nM}$ and $10 \mu \mathrm{g} / \mathrm{ml})$ for $72 \mathrm{~h}$. The total expression of Ki-67 was only significantly altered in MCF10A cells after SLC25A43 KD. For all assays, two experiments were performed in duplicates. The data are expressed as the means $\pm \mathrm{SD}$. ${ }^{*} \mathrm{P}<0.05$ and ${ }^{* *} \mathrm{P}<0.01$.

cycle (11-15). This was also confirmed in the BT-474 cells (Fig. 2D). Furthermore, KD of SLC25A43 leads to a significantly decreased G1 block at both 10 and $100 \mu \mathrm{g} / \mathrm{ml}$ of trastuzumab $(\mathrm{P}<0.05)$. This result suggests that KD of $S L C 25 A 43$ causes a reduced sensitivity to the inhibitory mechanisms of trastuzumab, which correlates with the results regarding the reduced inhibition of proliferation in SLC25A43 KD cells compared to control, following trastuzumab treatment (Fig. 1D). Exposure to trastuzumab in combination with paclitaxel resulted in reduced G2/M block compared to paclitaxel alone, however, the significant effect of $S L C 25 A 43 \mathrm{KD}$ on the G2/M block remained (Fig. 2C and D). These results demonstrate that KD of SLC25A43 in combination with drug exposure alters the cell cycle phase distribution in the breast cancer cell lines.

Ki-67 expression is altered by paclitaxel and epirubicin exposure. Loss of growth control, including aberrations in the mechanisms regulating the integrity of cell cycle progression, is a hallmark of cancer (27). Determining the expression of cell proliferation markers in tumour has therefore become 

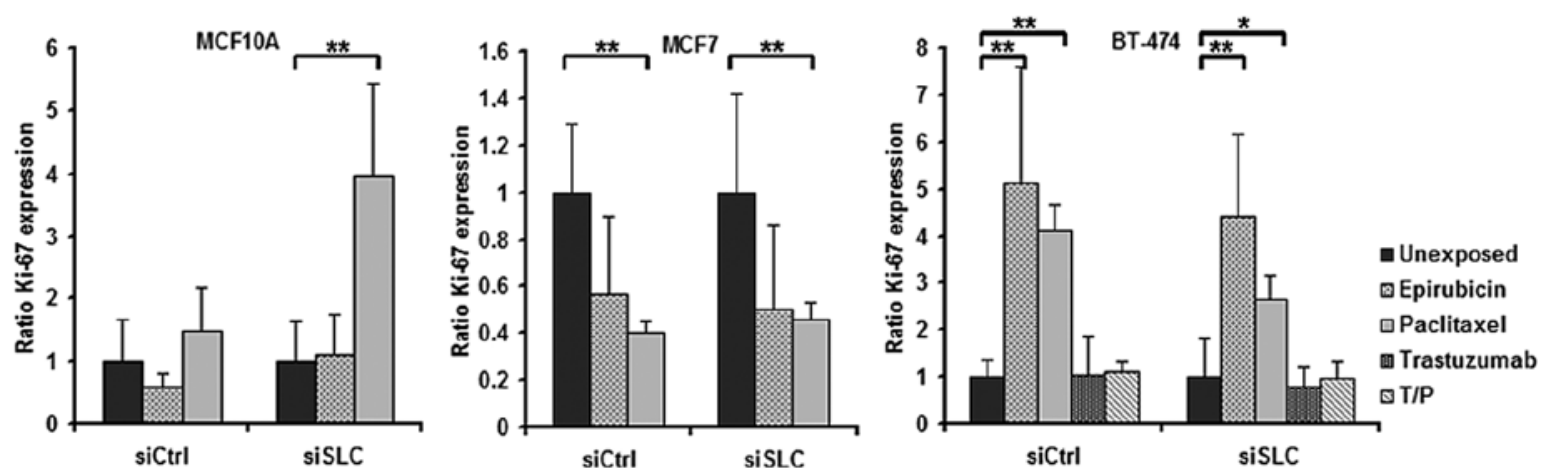

Figure 4. Ki-67 expression is altered after drug exposure. The graphs show the ratio of Ki-67 expression in MCF10A, MCF7 and BT-474 cells comparing unexposed and drug-exposed siCtrl or siSLC cells. The cells were exposed to $16 \mathrm{nM}$ paclitaxel or $2.5 \mu \mathrm{M}$ epirubicin for $72 \mathrm{~h}$. BT-474 cells were also exposed to $10 \mu \mathrm{g} / \mathrm{ml}$ trastuzumab or trastuzumab-paclitaxel double exposure (T/P) $(16 \mathrm{nM}$ and $10 \mu \mathrm{g} / \mathrm{ml})$ for $72 \mathrm{~h}$. In MCF10A cells, the ratio of Ki-67 expression was only significantly increased after paclitaxel exposure in siSLC. In MCF7 cells, the ratio of Ki-67 expression was significantly lower in both siCtrl and siSLC after paclitaxel exposure. In BT-474 cells, both epirubicin and paclitaxel significantly increased the ratio of Ki-67 expression in both siCtrl and siSLC. For all assays, two experiments were performed in duplicates. The data are expressed as the means $\pm \mathrm{SD}$. ${ }^{*} \mathrm{P}<0.05$ and ${ }^{* *} \mathrm{P}<0.01$.

increasingly important in the clinic $(31,32)$. One marker of interest is $\mathrm{Ki}-67$, a protein present in all phases of the cell cycle except in the G0 and the early G1 phase (33). In light of this and regarding the findings that KD of $S L C 25 A 43$ alters the cell cycle distribution upon drug exposure, we further investigated the effects of $S L C 25 A 43 \mathrm{KD}$ on the Ki-67 expression.

In MCF10A cells, KD of $S L C 25 A 43$ led to a decreased total percentage of $\mathrm{Ki}$-67-positive cells compared to siCtrl both in unexposed- and in epirubicin-exposed cells (Fig. 3). When exposing the cell lines to paclitaxel, the fraction of Ki-67positive cells was found not to be altered by KD of SLC25A43 when compared to siCtrl. However, paclitaxel was shown to influence the Ki-67 expression in all three cell lines (Fig. 4). In addition, exposure to epirubicin resulted in an increased fraction of Ki-67-positive BT-474 cells (Fig. 4).

As the mitochondrion has been demonstrated to impact the cell cycle regulation (20-26), we investigated if KD of $S L C 25 A 43$ in the three cell lines, when exposed to drugs, would contribute to changes in cell cycle regulation by analysis of p21 positivity. In our study, SLC25A43 KD and drug exposure did not influence the p21 positivity in any of the three cell lines (data not shown).

\section{Discussion}

The theory that mitochondria are important players of apoptosis, cell proliferation and cell cycle regulation (20-26) has expanded the field of cancer research, and it is now well established that mitochondrial dysfunction plays a key role in tumour development and response to therapy (27).

We previously demonstrated a common loss of the mitochondrial gene SLC25A43 in HER2-positive breast cancer (28). We further showed that HER2-positive breast tumours with a lower expression of SLC25A43 also have a lower $\mathrm{S}$ phase fraction. These previous findings led to the hypothesis that an altered mitochondrial function through a reduced expression of SLC25A43 may alter the efficacy of antitumour drugs in vitro.

Using siRNA, we investigated the effects of $S L C 25 A 43$ knockdown (KD) on drug cytotoxicity in immortalised mammary epithelial cells, HER2-negative, and HER2-positive breast cancer cells after exposure to different drugs. In our study, KD of SLC25A43 did not alter the viability following epirubicin exposure in any of the cell lines. This suggests that the mechanism of action of epirubicin cytotoxicity is, at least in part, independent of altered mitochondrial function due to $S L C 25 A 43 \mathrm{KD}$. Paclitaxel exerts is cytotoxic effects through other mechanisms, involving microtubule assembly, cell cycle arrest and the mitochondrial apoptotic pathway (34-36). Deregulations in this pathway and other mitochondrial functions have been suggested to induce paclitaxel resistance (36-38). In our experiments, exposure of the HER2-negative MCF7 cells and the HER2-positive BT-474 cells to paclitaxel after KD increased the viability of the cells compared to control. This change in viability was accompanied by an increased G2/M block in the exposed KD cells, indicating that further blocking of the cells in G2/M may be beneficial for cell survival. Tan et al (39) demonstrated that delaying cell entry into $\mathrm{M}$ phase conferred survival advantages following paclitaxel exposure in breast cancer cells. BT- 474 cells were further subjected to exposure to trastuzumab. KD of $S L C 25 A 43$ followed by exposure to trastuzumab resulted in decreased inhibitory effect on proliferation of the antibody and reduced the G0/G1 arrest. Trastuzumab is known to increase the association between $\mathrm{p} 27^{\mathrm{Kipl}}$ and CDK2 complexes resulting in induced G1 cell cycle arrest (11-15,40). Altered expression of these proteins has been connected with trastuzumab resistance (41). Our findings show that KD of $S L C 25 A 43$ reduces the G0/G1 arrest, possibly leading to the reduced inhibitory effect of trastuzumab in the HER2-positive BT-474 cells. When combining trastuzumab and paclitaxel to BT-474 cells, all survival and proliferative advantages due to $S L C 25 \mathrm{~A} 43$ KD previously observed are eradicated. This demonstrates the beneficial effect of dual exposure with cytostatic drugs and target therapies after $S L C 25 A 43 \mathrm{KD}$.

$\mathrm{Ki}-67$ is widely used as a proliferation marker in the clinic and it has also been shown to be a prognostic marker (31); however, studies investigating the predictive value of $\mathrm{Ki}-67$ for chemotherapy present inconclusive data (42-45). When investigating the Ki-67 expression in the cell lines after drug exposure, 
SLC25A43 KD was shown to influence the expression in the non-malignant MCF10A cells only after epirubicin exposure. However, both epirubicin and paclitaxel were found to alter the Ki-67 expression in the two breast cancer cell lines when compared to unexposed cells. MCF7 cells showed a decreased Ki-67 positivity while BT-474 cells showed an increased Ki-67 positivity following paclitaxel exposure. These findings may not be a direct result of the drug exposure; instead, it is more likely to be a secondary finding following the cell cycle arrest. It has previously been described in a clinical study that the Ki-67 expression was altered after chemotherapy, compared to before treatment, and a decreased expression was associated with reduced disease-free survival in rectal carcinoma (46). Also,p21 expression has been shown to be altered after treatment $(46,47)$. In our study, however, we were not able to assess any differences in p21 expression due to drug exposure. The clinical importance of an altered $\mathrm{Ki}-67$ and/or p21 expression after chemotherapy for patient outcome remains to be further elucidated.

Collectively, we have demonstrated that SLC25A43 alters the efficacy of paclitaxel through increased viability and increased G2/M arrest. A reduced expression of SLC25A43 also diminishes the antiproliferative effect of the target therapy trastuzumab. Our findings support the role of altered mitochondrial function in cancer and drug resistance.

\section{Acknowledgements}

We thank Edwin Reizer for the laboratory assistance and Professor Olle Stål for helpful discussions concerning the manuscript. This study was supported by grants from the Lion Cancer Foundation Sweden and the Research Committee at Örebro County Council.

\section{References}

1. Fossati R, Confalonieri C, Torri V, et al: Cytotoxic and hormonal treatment for metastatic breast cancer: a systematic review of published randomized trials involving 31,510 women. J Clin Oncol 16: 3439-3460, 1998.

2. Abal M, Andreu JM and Barasoain I: Taxanes: microtubule and centrosome targets, and cell cycle dependent mechanisms of action. Curr Cancer Drug Targets 3: 193-203, 2003.

3. Minotti G, Menna P, Salvatorelli E, Cairo G and Gianni L: Anthracyclines: molecular advances and pharmacologic developments in antitumor activity and cardiotoxicity. Pharmacol Rev 56: 185-229, 2004

4. Schiff PB, Fant J and Horwitz SB: Promotion of microtubule assembly in vitro by taxol. Nature 277: 665-667, 1979.

5. Frankel A, Buckman R and Kerbel RS: Abrogation of taxolinduced G2-M arrest and apoptosis in human ovarian cancer cells grown as multicellular tumor spheroids. Cancer Res 57: 2388-2393, 1997.

6. Sparano JA: Taxanes for breast cancer: an evidence-based review of randomized phase II and phase III trials. Clin Breast Cancer 1: $32-42,2000$

7. Cersosimo RJ and Hong WK: Epirubicin: a review of the pharmacology, clinical activity, and adverse effects of an adriamycin analogue. J Clin Oncol 4: 425-439, 1986.

8. Knoop AS, Knudsen H, Balslev E, et al: Retrospective analysis of topoisomerase IIa amplifications and deletions as predictive markers in primary breast cancer patients randomly assigned to cyclophosphamide, methotrexate, and fluorouracil or cyclophosphamide, epirubicin, and fluorouracil: Danish Breast Cancer Cooperative Group. J Clin Oncol 23: 7483-7490, 2005.

9. Early Breast Cancer Trialists' Collaborative Group (EBCTCG): Effects of chemotherapy and hormonal therapy for early breast cancer on recurrence and 15-year survival: an overview of the randomised trials. Lancet 365: 1687-1717, 2005.
10. Levine MN, Bramwell VH, Pritchard KI, et al: Randomized trial of intensive cyclophosphamide, epirubicin, and fluorouracil chemotherapy compared with cyclophosphamide, methotrexate, and fluorouracil in premenopausal women with node-positive breast cancer. National Cancer Institute of Canada Clinical Trials Group. J Clin Oncol 16: 2651-2658, 1998.

11. Baselga J, Albanell J, Molina MA and Arribas J: Mechanism of action of trastuzumab and scientific update. Semin Oncol 28: 4-11, 2001.

12. Sliwkowski MX, Lofgren JA, Lewis GD, Hotaling TE, Fendly BM and Fox JA: Nonclinical studies addressing the mechanism of action of trastuzumab (Herceptin). Semin Oncol 26: 60-70, 1999.

13. Mirza AM, Gysin S, Malek N, Nakayama K, Roberts JM and McMahon M: Cooperative regulation of the cell division cycle by the protein kinases RAF and AKT. Mol Cell Biol 24: 10868-10881,2004.

14. Mullany LK, Nelsen CJ, Hanse EA, et al: Akt-mediated liver growth promotes induction of cyclin $\mathrm{E}$ through a novel translational mechanism and a p21-mediated cell cycle arrest. J Biol Chem 282: 21244-21252, 2007.

15. Yakes FM, Chinratanalab W, Ritter CA, King W, Seelig S and Arteaga CL: Herceptin-induced inhibition of phosphatidylinositol-3 kinase and Akt is required for antibody-mediated effects on p27, cyclin D1, and antitumor action. Cancer Res 62: 4132-4141, 2002.

16. Montemurro F and Aglietta M: Incorporating trastuzumab into the neoadjuvant treatment of HER2-overexpressing breast cancer. Clin Breast Cancer 6: 77-80, 2005.

17. Montemurro F, Valabrega G and Aglietta M: Trastuzumab-based combination therapy for breast cancer. Expert Opin Pharmacother 5: 81-96, 2004.

18. Buzdar AU, Ibrahim NK, Francis D, et al: Significantly higher pathologic complete remission rate after neoadjuvant therapy with trastuzumab, paclitaxel, and epirubicin chemotherapy: results of a randomized trial in human epidermal growth factor receptor 2-positive operable breast cancer. J Clin Oncol 23: 3676-3685, 2005.

19. Piccart-Gebhart MJ, Procter M, Leyland-Jones B, et al: Trastuzumab after adjuvant chemotherapy in HER2-positive breast cancer. N Engl J Med 353: 1659-1672, 2005.

20. Finkel T and Hwang PM: The Krebs cycle meets the cell cycle: mitochondria and the G1-S transition. Proc Natl Acad Sci USA 106: 11825-11826, 2009

21. Detmer SA and Chan DC: Functions and dysfunctions of mitochondrial dynamics. Nat Rev Mol Cell Biol 8: 870-879, 2007.

22. Schieke SM, McCoy JP Jr and Finkel T: Coordination of mitochondrial bioenergetics with G1 phase cell cycle progression. Cell Cycle 7: 1782-1787, 2008.

23. Mandal S, Guptan P, Owusu-Ansah E and Banerjee U: Mitochondrial regulation of cell cycle progression during development as revealed by the tenured mutation in Drosophila. Dev Cell 9: 843-854, 2005.

24. Jones RG, Plas DR, Kubek S, et al: AMP-activated protein kinase induces a p53-dependent metabolic checkpoint. Mol Cell 18: 283-293, 2005.

25. Gwinn DM, Shackelford DB, Egan DF, et al: AMPK phosphorylation of raptor mediates a metabolic checkpoint. Mol Cell 30: 214-226, 2008

26. Owusu-Ansah E, Yavari A, Mandal S and Banerjee U: Distinct mitochondrial retrograde signals control the G1-S cell cycle checkpoint. Nat Genet 40: 356-361, 2008.

27. Hanahan D and Weinberg RA: Hallmarks of cancer: the next generation. Cell 144: 646-674, 2011.

28. Tina E, Malakkaran Lindqvist B, Gabrielson M, Lubovac Z, Wegman $\mathrm{P}$ and Wingren $\mathrm{S}$ : The mitochondrial transporter SLC25A43 is frequently deleted and may influence cell proliferation in HER2-positive breast tumors. BMC Cancer 12: 350, 2012.

29. Tina E, Prenkert M, Hoglund M, Paul C and Tidefelt U: Topoisomerase II $\alpha$ expression in acute myeloid leukaemia cells that survive after exposure to daunorubicin or ara-C. Oncol Rep 22: 1527-1531, 2009.

30. Lantz E, Cunningham I and Higa GM: Targeting HER 2 in breast cancer: overview of long-term experience. Int J Womens Health 1: 155-171, 2010.

31. Goldhirsch A, Ingle JN, Gelber RD, Coates AS, Thurlimann B and Senn HJ: Thresholds for therapies: highlights of the St Gallen International Expert Consensus on the primary therapy of early breast cancer 2009. Ann Oncol 20: 1319-1329, 2009. 
32. Butt AJ, Caldon CE, McNeil CM, Swarbrick A, Musgrove EA and Sutherland RL: Cell cycle machinery: links with genesis and treatment of breast cancer. Adv Exp Med Biol 630: 189-205, 2008.

33. Gerdes J, Lemke H, Baisch H, Wacker HH, Schwab U and Stein H: Cell cycle analysis of a cell proliferation-associated human nuclear antigen defined by the monoclonal antibody Ki-67. J Immunol 133: 1710-1715, 1984.

34. Tudor G, Aguilera A, Halverson DO, Laing ND and Sausville EA: Susceptibility to drug-induced apoptosis correlates with differential modulation of Bad, Bcl-2 and Bcl-xL protein levels. Cell Death Differ 7: 574-586, 2000.

35. Sunters A, Madureira PA, Pomeranz KM, et al: Paclitaxelinduced nuclear translocation of FOXO3a in breast cancer cells is mediated by c-Jun NH2-terminal kinase and Akt. Cancer Res 66: 212-220, 2006.

36. Kutuk $\mathrm{O}$ and Letai A: Alteration of the mitochondrial apoptotic pathway is key to acquired paclitaxel resistance and can be reversed by ABT-737. Cancer Res 68: 7985-7994, 2008.

37. Zhou M, Zhao Y, Ding Y, et al: Warburg effect in chemosensitivity: targeting lactate dehydrogenase-A re-sensitizes taxol-resistant cancer cells to taxol. Mol Cancer 9: 33, 2010.

38. Gogvadze V, Orrenius S and Zhivotovsky B: Mitochondria as targets for chemotherapy. Apoptosis 14: 624-640, 2009.

39. Tan M, Jing T, Lan KH, et al: Phosphorylation on tyrosine-15 of p34(Cdc2) by ErbB2 inhibits p34(Cdc2) activation and is involved in resistance to taxol-induced apoptosis. Mol Cell 9: 993-1004, 2002

40. Lane HA, Motoyama AB, Beuvink I and Hynes NE: Modulation of p27/Cdk2 complex formation through 4D5-mediated inhibition of HER2 receptor signaling. Ann Oncol 12 (Suppl 1): S21-S22, 2001.
41. Nahta R, Takahashi T, Ueno NT, Hung MC and Esteva FJ: $\mathrm{P} 27$ (kip1) down-regulation is associated with trastuzumab resistance in breast cancer cells. Cancer Res 64: 3981-3986, 2004.

42. Chang J, Ormerod M, Powles TJ, Allred DC, Ashley SE and Dowsett M: Apoptosis and proliferation as predictors of chemotherapy response in patients with breast carcinoma. Cancer 89: 2145-2152, 2000.

43. MacGrogan G, Mauriac L, Durand M, et al: Primary chemotherapy in breast invasive carcinoma: predictive value of the immunohistochemical detection of hormonal receptors, p53, c-erbB-2, MiB1, pS2 and GST pi. Br J Cancer 74: 1458-1465, 1996.

44. Colleoni M, Viale G, Zahrieh D, et al: Chemotherapy is more effective in patients with breast cancer not expressing steroid hormone receptors: a study of preoperative treatment. Clin Cancer Res 10: 6622-6628, 2004.

45. Rozan S, Vincent-Salomon A, Zafrani B, et al: No significant predictive value of c-erbB-2 or p53 expression regarding sensitivity to primary chemotherapy or radiotherapy in breast cancer. Int J Cancer 79: 27-33, 1998.

46. Rau B, Sturm I, Lage H, et al: Dynamic expression profile of p21WAF1/CIP1 and Ki-67 predicts survival in rectal carcinoma treated with preoperative radiochemotherapy. J Clin Oncol 21: 3391-3401, 2003

47. Pohl G, Rudas M, Taucher S, et al: Expression of cell cycle regulatory proteins in breast carcinomas before and after preoperative chemotherapy. Breast Cancer Res Treat 78: 97-103, 2003. 